\title{
An observational MRI study of methotrexate-treated children with acute lymphoblastic leukemia in remission and subtle cognitive decline
}

\author{
Ke Wei ${ }^{1,2} \wedge$, Yiwen Liang ${ }^{1}$, Binrang Yang ${ }^{3}$, Longping Liu ${ }^{1}$, Weiguo Cao ${ }^{1}$, Tian $\mathrm{Li}^{4}$, Rong Wang ${ }^{5}$ \\ ${ }^{1}$ Department of Radiology, Shenzhen Children's Hospital, Shenzhen, China; ${ }^{2}$ China Medical University, Shenyang, China; ${ }^{3}$ Center for Child Care \\ and Mental Health, Shenzhen Children's Hospital, Shenzhen, China; ${ }^{4}$ Department of Pathology and Cell Biology, Columbia University Medical \\ Center, New York, NY, USA; ${ }^{5}$ Department of Neurology, Zhongshan Hospital Xiamen University, Xiamen, China
}

Contributions: (I) Conception and design: K Wei, W Cao; (II) Administrative support: W Cao; (III) Provision of study materials or patients: K Wei, W Cao, B Yang, Y Liang, L Liu; (IV) Collection and assembly of data: K Wei, B Yang, L Liu; (V) Data analysis and interpretation: Y Liang, T Li, R Wang; (VI) Manuscript writing: All authors; (VII) Final approval of manuscript: All authors.

Correspondence to: Weiguo Cao. Department of Radiology, Shenzhen Children's Hospital, 7019 Yitian Road, Futian District, Shenzhen 518038, China. Email: weiguo.cao@126.com.

Background: For children diagnosed with acute lymphoblastic leukemia (ALL), methotrexate (MTX) treatment carries the risk of leukoencephalopathy and other treatment-related brain damage. However, earlier and more sensitive evaluation is needed to elucidate the specific effects of MTX treatment in this group. This study aimed to evaluate changes in brain metabolites, diffusion and anisotropy features, and cognitive performance in children with ALL after MTX treatment.

Methods: In this observational study conducted from December 2013 to December 2015, 30 children with ALL and 30 healthy children were recruited and evaluated using baseline magnetic resonance imaging (MRI), magnetic resonance spectroscopy (MRS), diffusion tensor imaging (DTI), and neurocognitive tests. After MTX treatment and ALL remission, the children with ALL underwent MR examination and neurocognitive tests again. Quantitative alterations of MR and cognitive test results from the baseline data were calculated.

Results: At baseline, the ALL group (age 6.9 \pm 3.3 years; 14 boys) and the healthy controls (age $6.0 \pm 3.1$ years, 14 boys) had comparable neurocognitive performance and MR results. After MTX treatment, 6.7\% (2/30) of children with ALL showed abnormalities on diffusion- and T1- and T2-weighted images. The $\mathrm{N}$-acetylaspartate/creatine and $\mathrm{N}$-acetylaspartate/choline values of children with ALL decreased, whereas their choline/creatine values increased significantly. The fractional anisotropy (FA) values decreased in the frontal lobe $(\mathrm{P}=0.03)$ and the genu of the corpus callosum $(\mathrm{P}=0.01)$. The FA values in the genu of the internal capsule $(\mathrm{P}=0.08)$, the occipital lobe $(\mathrm{P}=0.20)$ and the splenium of the corpus callosum $(\mathrm{P}=0.30)$ did not change from baseline. The apparent diffusion coefficient (ADC) values decreased in the frontal lobe $(\mathrm{P}=0.03)$. The ADC values in the genu of the corpus callosum $(\mathrm{P}=0.11)$, the genu of the internal capsule $(\mathrm{P}=0.93)$, and the occipital lobe $(\mathrm{P}=0.65)$ did not change from baseline. Due to the presence of outliers and the small sample, the ADC values in the splenium of the corpus callosum were discarded. Neurocognitive performance decreased slightly after MTX treatment, with noticeable declines in working memory and processing speed. Changes in FA values were positively correlated with the reduction in the $\mathrm{N}$-acetylaspartate/creatine ratio at the genu of the corpus callosum of children with ALL aged above 6 years.

Conclusions: MTX treatment causes subtle cognitive decline in children with ALL in remission and dramatically affects their brain metabolites, but changes in white matter diffusion features are limited to the

\footnotetext{
$\wedge$ ORCID: 0000-0002-1356-3705.
} 
frontal lobe and corpus callosum.

Keywords: Methotrexate; acute lymphoblastic leukemia (ALL); cognitive; magnetic resonance imaging (MRI)

Submitted Jul 23, 2021. Accepted for publication Dec 16, 2021; Published online: 17 Jan 2022.

doi: $10.21037 /$ qims-21-748

View this article at: https://dx.doi.org/10.21037/qims-21-748

\section{Introduction}

Magnetic resonance imaging (MRI) is a non-invasive, advanced diagnostic imaging technique with wide-ranging clinical and research applications (1). It not only sensitively detects lesions, but also serves as a powerful quantitative tool. MR spectroscopy (MRS) utilizes the $1 \mathrm{H}$ nucleus, as the prevalent isotope in most brain metabolites, as its spectral signal to directly measure the concentrations of brain metabolites, such as $\mathrm{N}$-acetylaspartate (NAA), creatine $(\mathrm{Cr})$, and choline (Cho). Another quantitative application of MRI is diffusion-tensor imaging (DTI), which estimates the diffusion rate of water molecules between cells to reflect in vivo microscopic interactions.

As a sensitive, non-invasive, in vivo qualitative and quantitative imaging technique, MRI has benefited many patients with mild-to-moderate as well as early neurologic or psychiatric conditions by providing critical information to help guide early therapeutic intervention. Methotrexate (MTX) is a powerful anticancer drug, but it can be neurotoxic when used as a standardized chemotherapy protocol, although symptoms may be transient in some cases (2). Neurotoxic side effects of MTX include leukoencephalopathy, which appears as transient, hyperintense regions on $\mathrm{T} 2$-weighted imaging (T2WI). In previous research, regularly scanning of patients with acute lymphoblastic leukemia (ALL) showed that leukoencephalopathy had a prevalence of between $17 \%$ and $87 \%$, and $15 \%$ to $83 \%$ of patients presented with leukoencephalopathy when scanned only after a central nervous system event (3). Survivors of childhood ALL have been shown to experience treatment-related complications, such as secondary malignancies or neurocognitive impairment, which impact their quality of life after remission (4). The underlying mechanism of MTX neurotoxicity involves alterations in folate physiology and homeostasis. Folate is essential for neurons and other cells of the central nervous system due to its critical roles in DNA and RNA synthesis, DNA methylation, and myelin maintenance (5). Sequential MRI examination facilitates the detection of leukoencephalopathy in all symptomatic patients with clinical neurotoxicity and in some asymptomatic patients after MTX treatment (2). During the acute phase of leukoencephalopathy, DTI reveals restricted diffusion of water molecules within the cerebral deep white matter, and some residual focus with increased signals on T2WI and fluid-attenuated inversion recovery (FLAIR) $(2,6)$. One study that used MRS to examine MTX-treated children with ALL revealed transient decreases in the NAA/ Cho and NAA/Cr values and temporary increases in the $\mathrm{Cho/Cr}$ values at 20 weeks after treatment (7).

For the present study, we hypothesized that MR examination (MRS and DTI) of children with ALL would allow for efficient monitoring of MTX neurotoxicity in patients who might be asymptomatic. We also hypothesized that changes in MR parameter sensitivity would correlate with neurocognitive performance and with changes in MRS and DTI parameters. MTX can cause structural damage to brain areas, mainly to white matter, especially in the frontal lobe and corpus callosum. The occipital lobe and internal capsule can also be involved, so these structures were selected for our study (8-11). This study aimed to evaluate changes in brain metabolites, diffusion and anisotropy features, and cognitive performance in children with ALL after MTX treatment.

We present the following article in accordance with the MDAR checklist and STROBE reporting checklist (available at https://qims.amegroups.com/article/ view/10.21037/qims-21-748/rc).

\section{Methods}

\section{Methods and materials}

This study was conducted in accordance with the Declaration of Helsinki (as revised in 2013). The study was approved by the Institutional Review Board of Shenzhen Children's Hospital, and informed consent for each of the patients was acquired from their parents or legal guardians. 


\section{Study participants}

Between December 2013 and December 2015, 30 children aged 2 to 14 years old with a first-time diagnosis of ALL were recruited randomly from Shenzhen Children's Hospital. All the children were treated with a standardized protocol of intravenous MTX, including induction and consolidation therapy. The standardized protocol and the clinical risk classification are detailed in the Appendix. All cases achieved complete remission of their malignancy. Children diagnosed with ALL who had an incomplete medical history or whose records were not available for evaluation were excluded from the study, as were those with any of the following complications: other hematological malignancies, neurologic disorders, congenital dysmorphism, or inborn errors of metabolism.

For the control group, 30 healthy children, who were matched to the ALL group by age, educational level, and sex at the individual level, were recruited from the hospital's neurological outpatient services department. These children had not taken any medications or received radiation that can cause conditions of functional neurologic disorders. Healthy children who did not have a complete evaluation and medical history were also ineligible to participate.

\section{MRI, MRS, and DTI acquisition and analysis}

The children in the ALL group underwent two MR examinations, at baseline and at 1 to 3 days after MTX treatment during ALL remission. The children were evaluated for remission at 46 days after receiving MTX. Human brain development is relatively stable after the first 24 months of life (12-14); hence, children under the age of 2 were excluded to minimize the effects of rapid maturation of white matter (15). Also, the interval between the two MR examinations was 46 to 49 days, and there were not many neurodevelopmental changes occurred in healthy individuals during such a short space of time. Therefore, healthy controls only underwent MR evaluation at baseline. Upon the completion of MTX treatment and confirmation of remission by bone marrow biopsy, children in the ALL group were scheduled for an MR examination within 3 days.

The MR images were acquired with a 3.0-T MRI scanner and workstation (Magnetom Skyra, Siemens Healthcare, Erlangen, Germany) using an 8-channel orthogonal head and neck coil. Children under 6 years of age who were unable to cooperate with the MR scan were sedated via anal administration of $0.5 \%$ chlorine hydrate.
The MR techniques used in this study included axial T1WI [repetition time/echo time (TR/TE) 1,800/40 ms; field of view (FOV) $24 \times 24 \mathrm{~cm}$; matrix $120 \times 93$; slice thickness $5 \mathrm{~mm}$ ], axial T2WI (TR/TE 2,300/120 ms), axial T2 FLAIR (TR/TE 9,000/135 ms), sagittal T1WI FLAIR (TR/TE 200/2.5 ms), multi-voxel 1H MRS, point resolved spectroscopy (TR/TE 1,500/35 ms; FOV $23 \times 23 \mathrm{~cm}$; matrix 64×64; number of layers 4-10; slice thickness $6 \mathrm{~mm}$ ), DTI [echo-planar imaging (EPI) sequences, b 1000, 20 dispersion directions; TR/TE $1,500 \mathrm{~ms} / 30 \mathrm{~ms}$; FOV $=12 \times 12 \mathrm{~cm}$; matrix $60 \times 60$; number of layers 15 ; slice thickness $5 \mathrm{~mm}$ ), and DWI [echo-planar imaging (EPI) sequences, TR/TE 200/2.5 ms; FOV $25 \times 25 \mathrm{~cm}$; matrix $100 \times 100$; slice thickness $5 \mathrm{~mm}$ ].

After processing, the workstation software syngoMMWP VE40B (Siemens AG, Munich, Germany) and the DTI analysis software Neuro 3D (Siemens AG, Munich, Germany) were used for image analysis. Five regions of interests (ROIs) were sampled with MRS, including the bilateral frontal white matter, the genu of the corpus callosum, and the genu of the bilateral internal capsule. In the frontal lobe, in order to avoid the influence of the frontal sinus and the frontal bone on the MRS examination, the location information area on $1 \mathrm{H}-\mathrm{MRS}$ was located in the bilateral posterior frontal lobes, including part of the basal ganglia, the bilateral anterior horns of the lateral ventricles, and the genu of the corpus callosum. Each ROI was approximately 3 to $8 \mathrm{~mm}^{3}$ in size. The ROIs of the MRS were measured repeatedly three times and then averaged. The volume was $30 \times 30 \times 20 \mathrm{~mm}^{3}$, and the voxel ROI volume size was $10 \times 10 \times 10 \mathrm{~mm}^{3}$. The NAA/Cr, Cho/Cr, and NAA/Cho values of the ROIs were calculated. The additional three ROIs, which included the bilateral occipital lobe and the splenium of the corpus callosum, were sampled by DTI. The size of each ROI on DTI was approximately 3 to $7 \mathrm{~mm}^{3}$. The apparent diffusion coefficient (ADC) and fractional anisotropy (FA) of the ROIs were calculated, and three-dimensional DTI maps were constructed. The average of the values measured from the bilateral sites represented the final data point.

The MR images, and MRS and DTI data were independently analyzed by two radiologists (W.C. and L.L., with 15 years and 6 years of post-training clinical experience, respectively), who were blinded to the participants' characteristics.

\section{Cognitive functional assessment of participants}

The cognitive function of the study participants was 
evaluated using the Wechsler Preschool and Primary Scale of Intelligence (WPPSI-III) test (2002) for children under 6 years of age (the under-6 group) and the Wechsler Intelligence Scale for Children (WISC-IV) test (2003) for children above 6 years of age (the over- 6 group). Two physicians (KW and BY, with 1 and 21 years of posttraining clinical experience, respectively) performed the cognitive assessments for each participant in cooperation, while blinded to their medical history. Both the healthy children and the children with ALL completed the baseline assessment before treatment, but only the children in the ALL group underwent additional evaluation, after MTX treatment and ALL remission.

\section{Statistical analysis}

Using SPSS software v. 20 (SPSS, Chicago, IL, USA), the baseline data (including data from cognitive tests and NAA/ $\mathrm{Cr}$, NAA/Cho, Cho/Cr, FA, and ADC values) of children with ALL and healthy controls were compared using the two-sample $t$-test. The baseline and post-treatment evaluation data of the children in the ALL group were compared using paired $t$-tests. A statistically significant difference was indicated by a $\mathrm{P}$ value of less than 0.05 . Data in this study are reported as the mean \pm standard deviation. Correlation analysis of MRS data (or DTI parameters) with cognitive scores was performed by using a linear regression model. The cognitive function of the participants was evaluated using the WPPSI-III test [2002] for children under 6 years of age and the WISC-IV test [2003] for children above 6 years of age. In all statistical analyses involving cognitive scores [including analyses of baseline data, post-treatment evaluation data, and the correlation of MRS data (DTI parameters) with cognitive scores], participants were categorized into older and younger groups using 6 years of age as the cut-off.

\section{Results}

\section{Participant characteristics}

Initially, 30 children with ALL (age $6.9 \pm 3.3$ years, 14 boys) who were scheduled for intravenous MTX treatment in Shenzhen Children's Hospital, China, were recruited. The intervals between the two MR examinations ranged from 46 to 49 days, with the median interval being 47 days. Participants' demographic features, baseline cognitive performance (before MTX treatment), and initial MRS and DTI evaluation parameters are summarized in Table 1. The symptoms experienced by the children with ALL are listed in Table 2. The demographic characteristics and cognitive performance scores of the 30 healthy children (age

Table 1 Demographic characteristics, baseline cognition, and MRI studies of methotrexate-treated children with acute lymphoblast leukemia and healthy controls

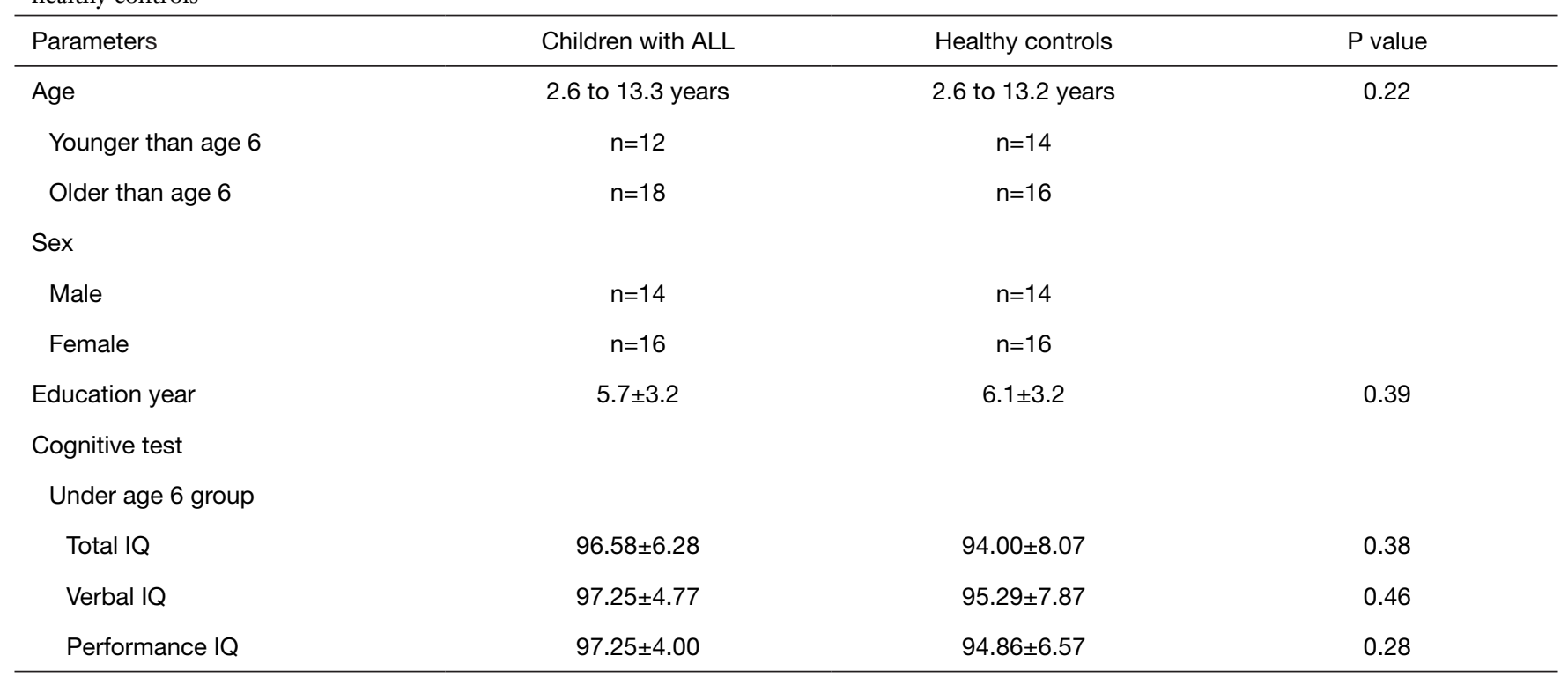

Table 1 (continued) 
Table 1 (continued)

\begin{tabular}{|c|c|c|c|}
\hline Parameters & Children with ALL & Healthy controls & $P$ value \\
\hline Total IQ & $97.39 \pm 3.93$ & $96.19 \pm 2.54$ & 0.30 \\
\hline Verbal comprehension & $88.28 \pm 3.03$ & $88.50 \pm 3.43$ & 0.84 \\
\hline Perceptual reasoning & $90.28 \pm 4.61$ & $92.19 \pm 2.20$ & 0.14 \\
\hline Processing speed & $99.39 \pm 3.47$ & $97.75 \pm 3.11$ & 0.16 \\
\hline \multicolumn{4}{|l|}{$\mathrm{NAA} / \mathrm{Cr}$} \\
\hline Frontal lobe & $1.88 \pm 0.56$ & $1.92 \pm 0.42$ & 0.75 \\
\hline Genu of the corpus callosum & $1.76 \pm 0.43$ & $1.80 \pm 0.34$ & 0.73 \\
\hline Frontal lobe & $1.17 \pm 0.20$ & $1.15 \pm 0.18$ & 0.65 \\
\hline Genu of the corpus callosum & $1.21 \pm 0.52$ & $1.13 \pm 0.34$ & 0.42 \\
\hline Genu of the internal capsule & $1.00 \pm 0.20$ & $1.00 \pm 0.20$ & 0.92 \\
\hline \multicolumn{4}{|l|}{ NAA/Cho } \\
\hline Frontal lobe & $1.66 \pm 0.55$ & $1.71 \pm 0.43$ & 0.67 \\
\hline Genu of the corpus callosum & $1.66 \pm 0.71$ & $1.71 \pm 0.52$ & 0.75 \\
\hline Genu of the internal capsule & $2.14 \pm 0.47$ & $2.11 \pm 0.39$ & 0.80 \\
\hline \multicolumn{4}{|l|}{ FA } \\
\hline \multicolumn{4}{|l|}{ ADC } \\
\hline Frontal lobe & $0.89 \pm 0.08$ & $1.09 \pm 1.12$ & 0.33 \\
\hline Genu of the corpus callosum & $0.83 \pm 0.11$ & $1.25 \pm 1.68$ & 0.18 \\
\hline Genu of the internal capsule & $0.86 \pm 0.07$ & $0.86 \pm 0.06$ & 0.81 \\
\hline Occipital lobe & $0.75 \pm 0.06$ & $0.77 \pm 0.06$ & 0.22 \\
\hline Splenium of the corpus callosum & $0.77 \pm 0.04$ & $0.78 \pm 0.05$ & 0.24 \\
\hline
\end{tabular}

Data are presented as the mean \pm standard deviation. ALL, acute lymphoblastic leukemia; IQ, intelligence quotient; NAA, $N$-acetylaspartate; Cho, choline; $\mathrm{Cr}$, creatine; FA, fractional anisotropy; ADC, apparent diffusion coefficient. 
Table 2 Signs and symptoms of children with acute lymphoblastic leukemia (ALL), at diagnosis, as number $(\mathrm{N})$ and percentage (\%)

\begin{tabular}{lcc}
\hline Symptoms & N & $\%$ \\
\hline Lymphadenectasis & 9 & 30 \\
Skeleton pain & 4 & 13.33 \\
Fever & 19 & 63.33 \\
Hepatosplenomegaly & 30 & 100 \\
Petechial or mucosal bleeding & 20 & 66.67 \\
\hline
\end{tabular}

$6.0 \pm 3.1$ years, 14 boys) recruited from the hospital's neurological outpatient services department were comparable to those of the ALL group at baseline. In the under-6 group (12 in ALL group and 14 in control group), 7 children in the ALL group and 9 healthy controls were sedated. Neither the children with ALL nor the healthy controls had any abnormal T1WI, T2WI, or restricted water diffusion signals. No significant differences were observed in the characteristics [age, sex, educational years, and intelligence quotient (IQ)], initial MRS or DTI parameters, or cognitive scores of the two groups (Table 1).

\section{Abnormal findings on MRI after MTX treatment}

After the completion of the MTX regimen, all 30 children with ALL achieved hematological remission. One child had an abnormal hypointensity signal on T1WI, coupled with hyperintensity on T2WI and a restricted water diffusion signal on DWI. Another patient had abnormal hyperintensity on T2WI and associated hyperintensity on DWI (Figure 1A-1H). Seven children (7/30, 23\%) displayed mild brain atrophy, physiological manifestations of which included widening split-brain, reduced brain volume, and dilated ventricles (Figure 2A,2B). However, using threedimensional DTI stereoscopical technology, the white matter fiber bundles were digitally reconstructed. No gross abnormalities in the shape or volume of the fiber bundles were observed after MTX treatment.

\section{Alteration of brain metabolites on MRS after MTX treatment}

MRS showed that MTX treatment had altered the brain metabolite ratios of children with ALL (Figure $3 A-3 D$ ). In the frontal lobe, the NAA/Cr ratio decreased by $43.6 \%$ $(1.88 \pm 0.56$ vs. $1.06 \pm 0.12 ; \mathrm{P}<0.001)$, the $\mathrm{Cho} / \mathrm{Cr}$ ratio increased by $22.2 \%(1.17 \pm 0.20$ vs. $1.43 \pm 0.18 ; \mathrm{P}<0.001)$, and the NAA/Cho ratio decreased by $54.8 \%(1.66 \pm 0.55 \mathrm{vs}$. $0.75 \pm 0.14 ; \mathrm{P}<0.001)$. In the genu of the corpus callosum, the NAA/Cr ratio decreased by $42.0 \%(1.76 \pm 0.43 v s$. $1.02 \pm 0.21 ; \mathrm{P}<0.001)$, the $\mathrm{Cho} / \mathrm{Cr}$ ratio increased by $20.7 \%$ $(1.21 \pm 0.52$ vs. $1.46 \pm 0.18 ; \mathrm{P}=0.01)$, and the $\mathrm{NAA} /$ Cho ratio decreased by $57.8 \%(1.66 \pm 0.71$ vs. $0.70 \pm 0.15 ; \mathrm{P}<0.001)$. In the genu of the internal capsule, the $\mathrm{NAA} / \mathrm{Cr}$ ratio decreased by $47.7 \%(2.14 \pm 0.60$ vs. $1.12 \pm 0.14 ; \mathrm{P}<0.001)$, the Cho/Cr ratio increased by $52.0 \%(1.00 \pm 0.20$ vs. $1.52 \pm 0.21$; $\mathrm{P}<0.001$ ), and the NAA/Cho ratio decreased by $64.5 \%$ $(2.14 \pm 0.47$ vs. $0.76 \pm 0.16 ; \mathrm{P}<0.001)$. In all three areas, all the neurochemical ratios (NAA, Cho, and Cr) changed significantly (Figure 3D). The differences between baseline and after treatment were larger in the genu of the internal capsule than in the other two areas.

\section{Alteration of DTI parameters after MTX treatment}

To assess the impact of MTX on the local diffusion features of white matter, the difference in the FA values after treatment was calculated for the five areas (Figure $4 A$ ). In the frontal lobe, the FA values after treatment decreased by $10.8 \%(0.38 \pm 0.06$ vs. $0.34 \pm 0.08 ; \mathrm{P}=0.03)$, and the ADC values decreased by $4.7 \%(0.89 \pm 0.08$ vs. $0.85 \pm 0.07$; $\mathrm{P}=0.03)$. In the genu of the corpus callosum, the FA values decreased by $13.2 \%(0.74 \pm 0.15$ vs. $0.64 \pm 0.15 ; \mathrm{P}=0.01)$, but the ADC values $(0.83 \pm 0.11$ vs. $0.77 \pm 0.12 ; \mathrm{P}=0.11) \mathrm{did}$ not change. In the genu of the internal capsule, neither the FA $(0.42 \pm 0.13$ vs. $0.37 \pm 0.14 ; \mathrm{P}=0.08)$ nor the $\mathrm{ADC}$ $(0.86 \pm 0.07$ vs. $0.85 \pm 0.05 ; \mathrm{P}=0.93)$ values showed a change from baseline. The FA values in the splenium of the corpus callosum $(0.59 \pm 0.05$ vs. $0.56 \pm 0.15 ; \mathrm{P}=0.30)$ and the occipital lobe $(0.60 \pm 0.09$ vs. $0.57 \pm 0.07 ; \mathrm{P}=0.20)$ did not change from baseline, nor did the ADC values in the occipital lobe ( $0.75 \pm 0.06$ vs. $0.76 \pm 0.06 ; \mathrm{P}=0.65)$. Due to the presence of outliers and the small sample, the ADC values in the splenium of the corpus callosum were discarded (Figure $4 B, 4 C)$.

\section{Cognitive decline of children with ALL after MTX treatment}

MTX treatment impaired the cognitive performance of children with ALL. After treatment, the under-6 group exhibited lower scores for total intelligence quotient (IQ) $(-6.6 \%, 96.58 \pm 6.28$ vs. $90.25 \pm 7.12 ; \mathrm{P}=0.002)$, verbal IQ $(-6.9 \%, 97.25 \pm 4.77$ vs. $90.58 \pm 4.78 ; \mathrm{P}=0.003)$, and 

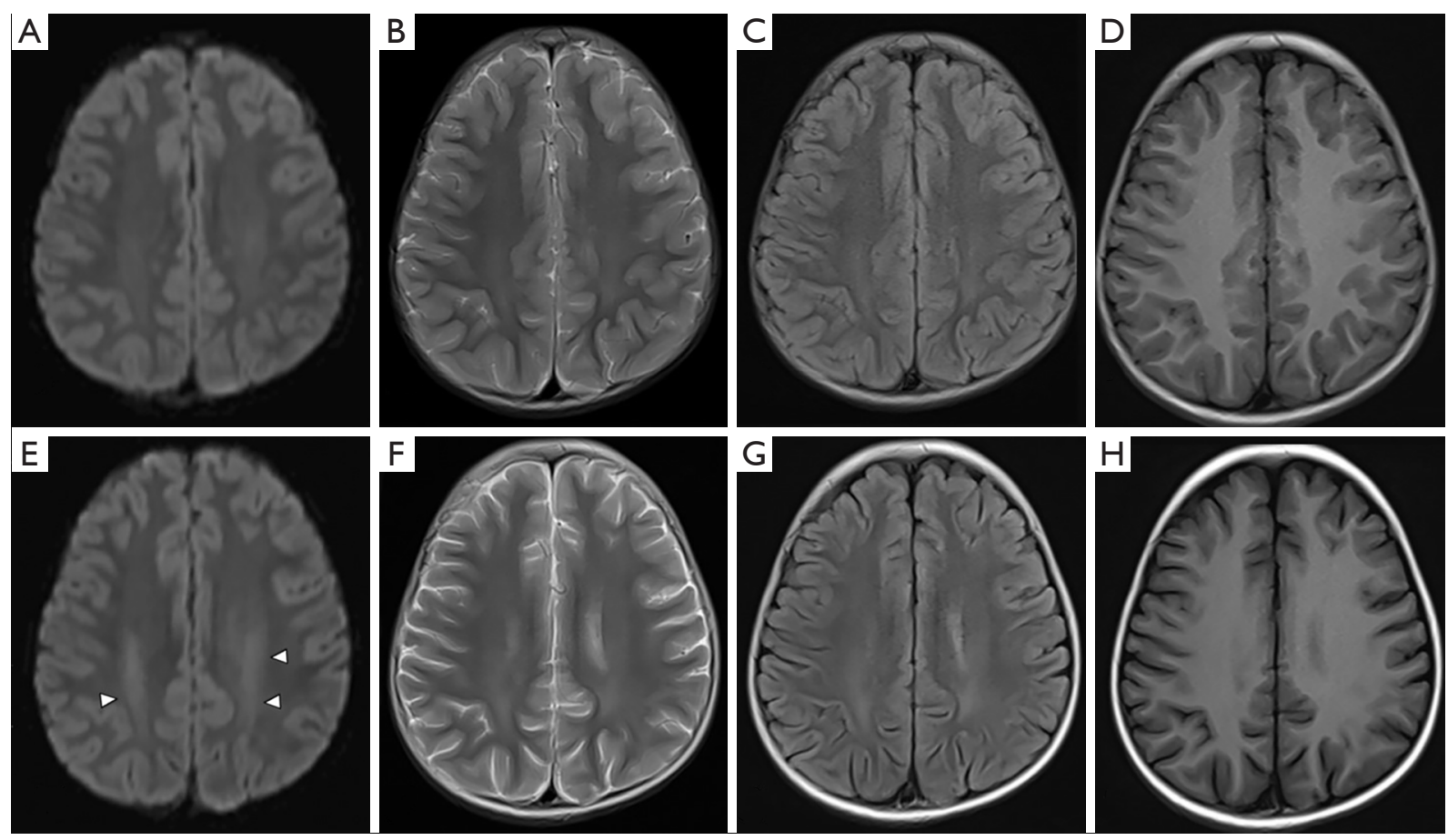

Figure 1 MR non-contrast images of a 2-year-old girl with ALL [repetition time/echo time (TR/TE) 1,800/40 ms] at the baseline and after MTX treatment. (A) Normal diffusion-weighted image at baseline; (B) T2-weighted image at baseline; (C) T2-weighted FLAIR image at baseline; (D) T1-weighted images at baseline; (E) diffusion-weighted image after treatment showing restricted water diffusion and hyperintensity in the deep white matter of both cerebral hemispheres (arrowheads); (F) T2-weighted image after treatment showing the hyperintensity in the deep white matter of both cerebral hemispheres; (G) T2-weighted fluid-attenuated inversion recovery image showing the hyperintensity in the deep white matter of both cerebral hemispheres; (H) T1-weighted image showing no abnormal intensity. MR, magnetic resonance; ALL, acute lymphoblast leukemia; MTX, methotrexate; FLAIR, fluid-attenuated inversion recovery.
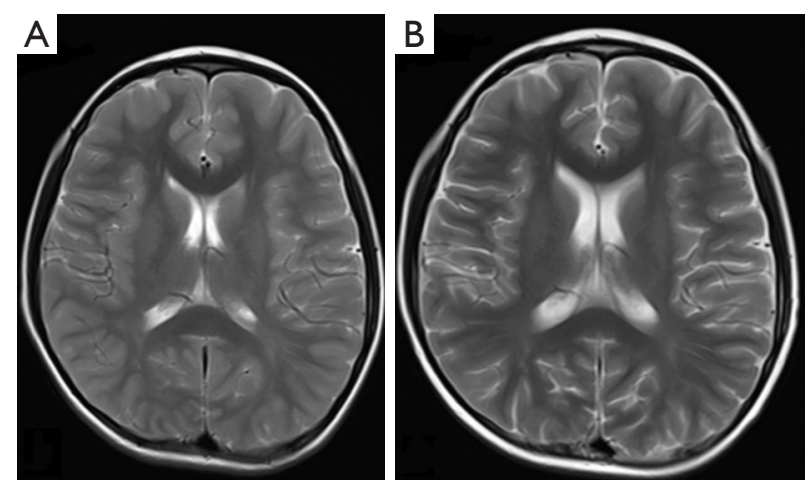

Figure 2 MR T2-weighted images of a 5-year-old boy with acute lymphoblast leukemia [ALL; repetition TR/T 1,800/40 ms] at baseline and after MTX treatment. (A) Normal T2-weighted image at baseline; (B) T2-weighted image after treatment, showing part of the brain fissure widened and deepened, and the bilateral lateral ventricles dilated. MR, magnetic resonance; TR/T, time/ echo time; MTX, methotrexate. performance IQ $(-7.0 \%, 97.25 \pm 4.00$ vs. $90.42 \pm 3.20$; $\mathrm{P}<0.001)$, while the over-6 group displayed lower scores for total IQ $(-3.1 \%, 97.39 \pm 3.93$ vs. $94.39 \pm 6.80 ; \mathrm{P}=0.02)$, perceptual reasoning $(-3.2 \%, 90.28 \pm 4.61$ vs. $87.39 \pm 3.16$; $\mathrm{P}=0.02)$, working memory $(-9.6 \%, 102.22 \pm 4.83$ vs. $92.44 \pm 7.74 ; \mathrm{P}<0.001)$, and processing speed $(-7.7 \%$, $99.39 \pm 3.47$ vs. $91.72 \pm 6.03 ; \mathrm{P}<0.001)$. However, no difference was observed in verbal comprehension scores $(88.28 \pm 3.03$ vs. $87.56 \pm 3.20 ; \mathrm{P}=0.30)$ in the over-6 group (Figure $5 A$ ). These results indicated that MTX treatment led to a prominent reduction in cognitive scores for working memory and processing speed.

\section{Relationships of altered MRS and DTI parameters, and cognitive decline in children with ALL}

Next, the relationships between alterations in brain metabolite ratios and DTI parameters and cognitive decline 

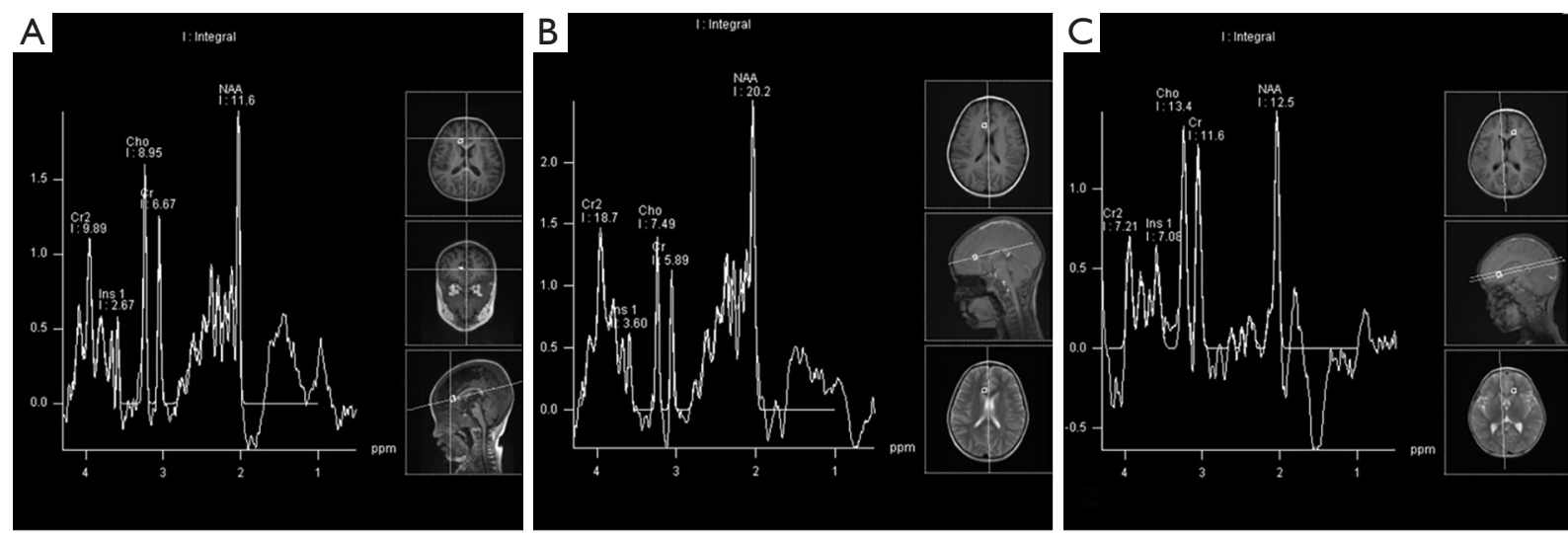

D

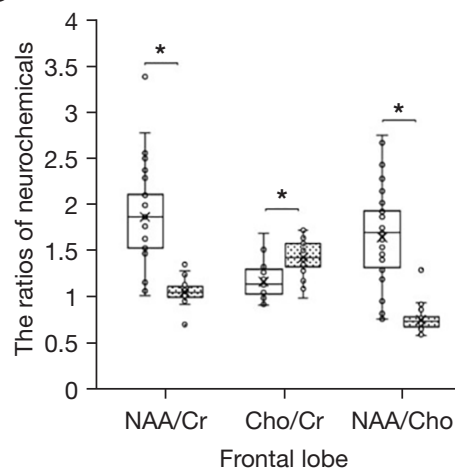

The ratios of NAA/Cr, Cho/Cr, and NAA/Cho at three areas

$\square A L L$ children before treatment $\quad \square$ ALL children after treatment

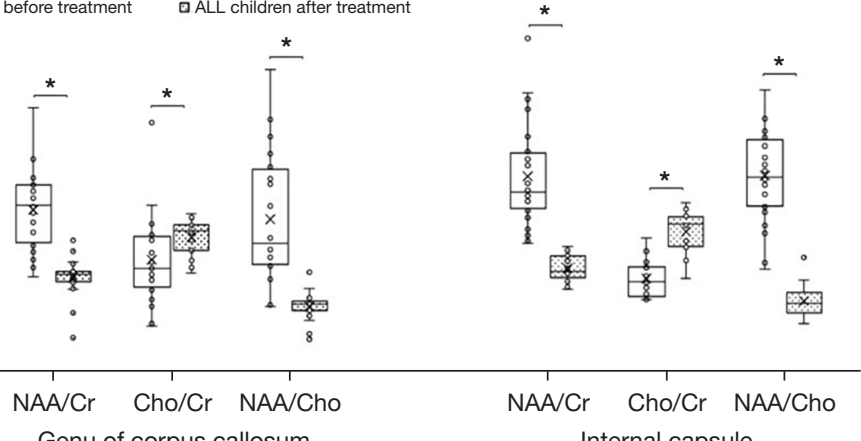

Figure 3 MRS image samples and statistical analysis of the NAA/Cr, Cho/Cr, and NAA/Cho ratios of children with ALL between the baseline and after MTX treatment. (A) MRS image acquired from a normal child; (B) MRS image acquired from a child with ALL at baseline; (C) MRS image acquired from a child with ALL after MTX treatment; (D) the NAA/Cr, and NAA/Cho ratios in the frontal lobe, the genu of the corpus callosum, and the internal capsule all decreased significantly after MTX treatment (n=30), while the Cho/Cr ratios in three areas increased significantly after MTX treatment $(n=30) .{ }^{*}$, indicates that the difference between the baseline and post-treatment was statistically significant. MRS, magnetic resonance spectroscopy; ALL, acute lymphoblast leukemia; MTX, methotrexate; NAA, $\mathrm{N}$-acetylaspartate, Cho, choline, Cr, creatine.

were further explored. Separate multiple linear regression models were calculated for the over- 6 group and the under- 6 group to predict cognitive decline based on the alterations in brain metabolite ratios and DTI parameters. The regression was not statistically significant. However, a relationship between metabolite ratios and DTI parameters was encountered. After MTX treatment, the change in the FA values $(\triangle \mathrm{FA})$ at the genu of the corpus callosum in the over-6 group was correlated with the change in the local NAA/Cr ratio $(\triangle \mathrm{NAA} / \mathrm{Cr} ; \mathrm{r}=0.51 ; \mathrm{P}=0.04)$ (Figure $5 B)$, whereas no obvious correlation was found for the under-6 group. All correlation analyses were corrected by the Benjamini and Hochberg (BH) false discovery rate (FDR) method.

\section{Discussion}

ALL is the most prevalent childhood malignancy, accounting for $26.8 \%$ of childhood cancers diagnosed in developed countries (16). Brain neurotoxicity in ALL includes cerebrovascular events, such as infarction and haemorrhage, or intracranial cancer cell infiltration $(17,18)$. In the past decade, therapeutic strategies have improved the survival rate among patients with ALL (19). However, neurocognitive development in survivors treated with MTX should be carefully monitored due to the risk of acute and chronic neurotoxicity caused by this drug (20). In the present study, MTX-treated children with ALL showed subtle cognitive decline (decreased cognitive scores and impaired working memory and processing 

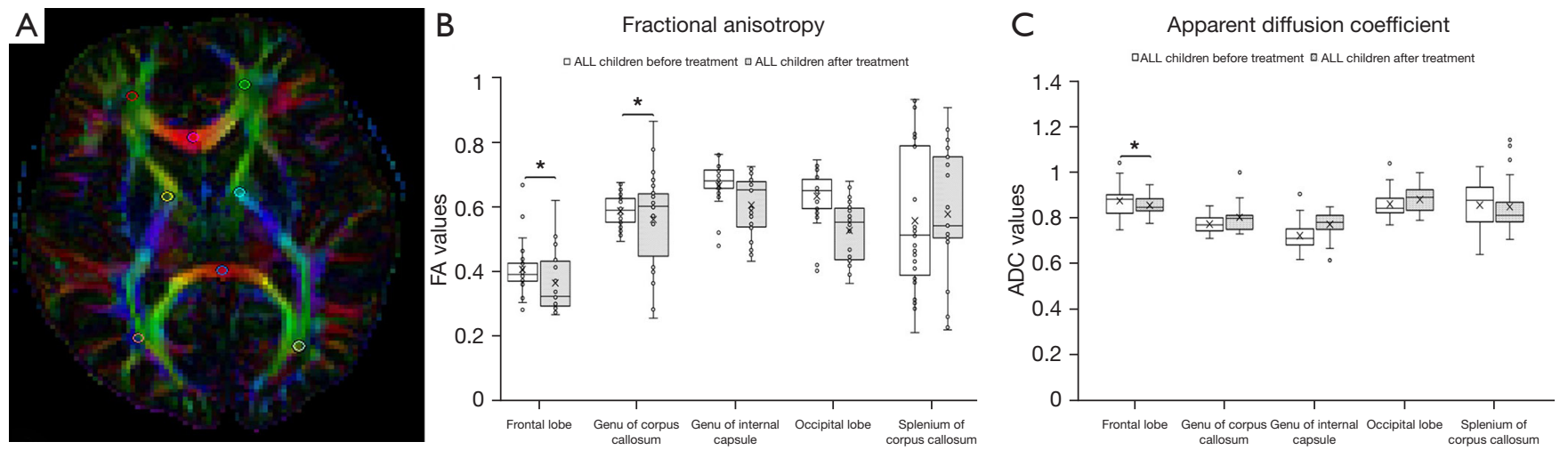

Figure 4 ROIs on DTI and statistical analysis of the FA and ADC values of children with ALL. (A) The eight ROIs selected for DTI parameter evaluation: left and right frontal lobes, genu of the corpus callosum, left and right genu of the internal capsule, left and right occipital lobe, and the splenium of the corpus callosum. (B) The FA values of the frontal lobe, genu of corpus callosum, genu of internal capsule, occipital lobe, and splenium of corpus callosum of the children with ALL at baseline and after MTX treatment (n=30). After treatment, the FA values in the frontal lobe $(\mathrm{P}=0.03)$ and the genu of the corpus callosum $(\mathrm{P}=0.01)$ were reduced compared to those at baseline. (C) The ADC values in the frontal lobe decreased $(\mathrm{P}=0.03)$ after treatment $(\mathrm{n}=30)$. Due to the presence of outliers and the small sample, the $\mathrm{ADC}$ values in the splenium of the corpus callosum were discarded. *, indicates that the difference between the measurements at baseline and after treatment was significant. ROI, region of interest; FA, fractional anisotropy; ADC, apparent diffusion coefficient; DTI, diffusion tensor imaging; ALL, acute lymphoblast leukemia.

speed). Although not all the children with ALL had acute symptoms of neurotoxicity (such as confusion, seizure, or muscle weakness), their neurocognitive decline did indicate neuronal or axonal injury related to MTX treatment. The MRI results revealed bilateral abnormalities in deep white matter on T1WI or T2WI in 2 children (7\%). The MRS assessment further revealed considerable changes (20-64\%) between baseline and after treatment in ALL, when compared to cognitive performance (3-9\%) or DTI parameters (4-13\%). Substantial changes in brain metabolites were found widely within the frontal lobe, the genu of the corpus callosum, and the internal capsule. The results of DTI showed a different pattern of alteration, with a significant decrease in both the FA and ADC values appearing only in the frontal lobe. The white matter of the frontal lobe was abnormal in both cellular metabolism and axonal integrity. Therefore, MTX treatment may have interfered with the microscopic integrity or connectivity of white matter in the frontal lobe.

In the present study, abnormalities detected in the deep white matter of the bilateral cerebral hemispheres on T1WI and T2WI were consistent with the MR findings of leukoencephalopathy reported in another study (2). And these abnormalities in MR may resolved 1 year after treatment (7). White matter injury is one of the major treatment-induced brain defects seen in survivors of childhood ALL (4). Our results revealed that 7 MTXtreated children had mild brain atrophy, which was also reported in another study, along with decreased grey and white matter volumes in cortical and several subcortical brain regions after chemotherapy (4). The MTX-induced changes in brain metabolite ratios were consistent with those observed in Chu's study (7). Furthermore, the NAA content increased logarithmically with age, while the Cho content decreased logarithmically with age $(21,22)$. This may indicate that, at a younger age, robust neuronal and axonal development and myelin sheathing may be more vulnerable to MTX neurotoxicity (23).

Creatine is a reminder of energy metabolism in brain tissue, and its kurtosis is stable compared with peaks of other brain metabolites. This experiment therefore chose creatine as the reference peak. NAA is related to the synthesis of myelin in the developing brains of children, and can also repair the axons of the mature brain (24). Therefore, the decrease of NAA in the frontal lobe, the genu of the internal capsule, and the genu of the corpus callosum may have been indicative of a reduction in neurons, or some demyelination changes occurring. Considering the possible threshold effect, the decrease of NAA that may have occurred it has not been shown on the baseline MR 


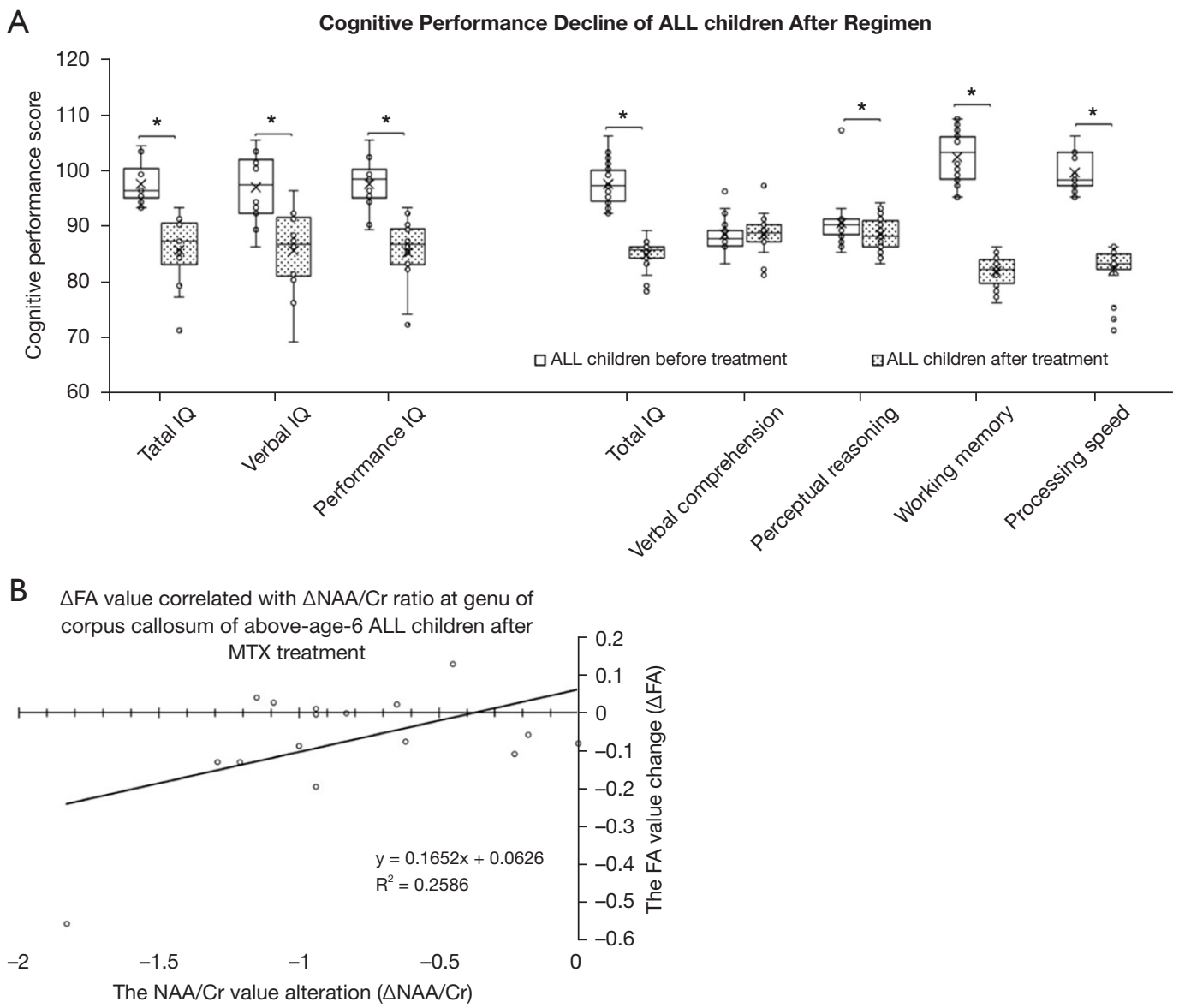

Figure 5 The cognitive performance of children with ALL and the correlations of brain metabolite ratios and DTI parameters. (A) The children with ALL displayed significantly reduced cognitive test scores after MTX treatment. The total $(\mathrm{P}=0.002)$, verbal $(\mathrm{P}=0.003)$, and performance IQ scores $(\mathrm{P}<0.001)$ all decreased in children aged under 6 years. In children aged over 6 years, the total IQ $(\mathrm{P}=0.02)$, perceptual reasoning $(\mathrm{P}=0.02)$, working memory $(\mathrm{P}<0.001)$, and processing speed $(\mathrm{P}<0.001)$ scores all decreased, but no change was observed in the verbal comprehension score. (B) In the over-6 group $(\mathrm{n}=18)$, the reduction in FA value $(\Delta \mathrm{FA})$ after MTX treatment was positively correlated $(\mathrm{r}=0.51, \mathrm{P}=0.04)$ with the $\mathrm{NAA} / \mathrm{Cr}$ ratio reduction $(\triangle \mathrm{NAA} / \mathrm{Cr})$ in the genu of the corpus callosum. *, indicates that the difference between the measurements at baseline and after treatment was significant. FA, fractional anisotropy; ADC, apparent diffusion coefficient; DTI, diffusion tensor imaging; ALL, acute lymphoblast leukemia; NAA, N-acetylaspartate; Cr, creatine; IQ, intelligence quotient.

at present. Furthermore, the metabolism of choline main reminds the metabolism of phospholipid, this may also reflect the degradation of myelin products and the integrity of myelination (25). An increase in Cho kurtosis may indicate demyelinating changes in the brain and gliosis. In our study, the increases in Cho in the frontal lobe, the genu of the internal capsule, and the genu of the corpus callosum may have prompted the increase of myelin degradation products in these regions and damage to the myelin sheath. According to a logarithmic curve, the content of NAA increased with age, while that of Cho decreased, which contradicts the MRS results of our study. This may serve as further evidence that the changes are caused by treatment. Our findings also revealed that the FA values significantly decreased at the genu of the corpus callosum after MTX treatment and that the reduction in the FA values was also correlated with a decrease in the local NAA/Cr ratio.

Microstructural injury of the genu of the corpus callosum might partially cause cognitive decline in the processing speed of children with ALL who receive MTX treatment (26). The corpus callosum is the largest white matter fiber bundle connecting the two cerebral hemispheres. The 
integrity of the corpus callosum is essential to children's cognitive development (27). During brain development and maturation, nerve fiber myelination gradually increases the cellular content of the brain and decreases its water content $(28,29)$. The developmental changes in the FA and ADC values observed in Salat et al.'s study were consistent with decreasing diffusivity and increasing anisotropy (30). Changes in DTI parameters may indicate that the frontal lobe is the brain part that is most vulnerable to MTX neurotoxicity (31). At present, researchers have differing opinions regarding the brain changes on DTI in various periods after chemotherapy for ALL. Some studies $(32,33)$ have found that the FA values of brain tissue decrease, whereas others (34) have reported that these values increase. The cause of the above-mentioned contradictions is still unclear. One explanation may be that the integrity of the white matter myelin sheath is affected differently in different parts of the brain. In the future, more comprehensive and time-point research is required to further explore the changes in FA and ADC values in MTX-treated ALL.

Our study has the following limitations. Firstly, the sample sizes $(n=30)$ of the ALL and healthy control groups in this study were small. Secondly, the first MR examination for the children with ALL was scheduled for 1 to 3 days after complete remission was confirmed. The second MR examination was scheduled for at least 46 days after the completion of MTX treatment, by which point some asymptomatic children may have recovered from MTXrelated leukoencephalopathy and become negative on T1 WI and T2 WI $(3,35)$. After intensive treatment, there might have impacted on the children's energy levels or psychological state, the effect of this on their cognitive scores cannot be overlooked. Also, the influence of neurodevelopment cannot be ruled out on our results. The diffusion tensor has been shown to fail in regions containing several fiber populations with distinct orientations. Although alternative models have been suggested, each of these has inherent limitations, and our study is no exception. The evaluation of brain atrophy and three-dimensional tele-immersion was based on the radiologists' subjective judgement, without an objective quantitative protocol.

In conclusion, ${ }^{1} \mathrm{H}$ MRS and DTI are able to detect changes associated with cognitive outcomes in children with ALL after MTX treatment. MRS and DTI might be useful for monitoring the effects of MTX neurotoxicity on brain metabolism and the microstructure of white matter fibers. Baseline MR evaluation, MRS, and DTI have the potential to be effective methods for detecting leukoencephalopathy in children with ALL after MTX treatment and for efficiently monitoring changes in neuroanatomic structure and brain metabolism in this group. The prospects of longterm MR examination follow-up of children with MTXtreated ALL are favourable. For research purposes, in the future, with multiple scoring methods, a systematic, comprehensive, and large sample-sized clinical study protocol will be desirable.

\section{Acknowledgments}

Funding: This work was supported by the Sanming Project of Medicine in Shenzhen (No. SZSM202011005).

\section{Footnote}

Reporting Checklist: The authors have completed the MDAR checklist and STROBE reporting checklist. Available at https://qims.amegroups.com/article/view/10.21037/qims$21-748 / \mathrm{rc}$

Conflicts of Interest: All authors have completed the ICMJE uniform disclosure form (available at https:// qims.amegroups.com/article/view/10.21037/qims-21$748 /$ coif). WC reports having received grants from the Sanming Project of Medicine in Shenzhen (Grant No. SZSM202011005) during the conduct of the study. The other authors have no conflicts of interest to declare.

Ethical Statement: The authors are accountable for all aspects of the work in ensuring that questions related to the accuracy or integrity of any part of the work are appropriately investigated and resolved. The study was conducted in accordance with the Declaration of Helsinki (as revised in 2013). The study was approved by the institutional review board of Shenzhen Children's Hospital and informed consent was acquired from all the patients' parents or legal guardians.

Open Access Statement: This is an Open Access article distributed in accordance with the Creative Commons Attribution-NonCommercial-NoDerivs 4.0 International License (CC BY-NC-ND 4.0), which permits the noncommercial replication and distribution of the article with the strict proviso that no changes or edits are made and the original work is properly cited (including links to both the formal publication through the relevant DOI and the license). See: https://creativecommons.org/licenses/by-nc-nd/4.0/. 


\section{References}

1. Govindaraju V, Young K, Maudsley AA. Proton NMR chemical shifts and coupling constants for brain metabolites. NMR Biomed 2000;13:129-53.

2. Bhojwani D, Sabin ND, Pei D, Yang JJ, Khan RB, Panetta JC, Krull KR, Inaba H, Rubnitz JE, Metzger ML, Howard SC, Ribeiro RC, Cheng C, Reddick WE, Jeha S, Sandlund JT, Evans WE, Pui CH, Relling MV. Methotrexateinduced neurotoxicity and leukoencephalopathy in childhood acute lymphoblastic leukemia. J Clin Oncol 2014;32:949-59.

3. Schroyen G, Meylaers M, Deprez S, Blommaert J, Smeets A, Jacobs S, Sunaert S, Sleurs C, Uyttebroeck A. Prevalence of leukoencephalopathy and its potential cogni-tive sequelae in cancer patients. J Chemother 2020;32:327-43.

4. Zhou C, Zhuang Y, Lin X, Michelson AD, Zhang A. Changes in neurocognitive function and central nervous system structure in childhood acute lymphoblastic leukaemia survivors after treatment: a meta-analysis. Br J Haematol 2020;188:945-61.

5. Cole PD, Kamen BA. Delayed neurotoxicity associated with therapy for children with acute lymphoblastic leukemia. Ment Retard Dev Disabil Res Rev 2006;12:174-83.

6. Inaba $\mathrm{H}$, Khan $\mathrm{RB}$, Laningham $\mathrm{FH}$, Crews KR, Pui CH, Daw NC. Clinical and radi-ological characteristics of methotrexate-induced acute encephalopathy in pediatric patients with cancer. Ann Oncol 2008;19:178-84.

7. Chu WC, Chik KW, Chan YL, Yeung DK, Roebuck DJ, Howard RG, Li CK, Me-treweli C. White matter and cerebral metabolite changes in children undergoing treatment for acute lymphoblastic leukemia: longitudinal study with MR imaging and $1 \mathrm{H}$ MR spectroscopy. Radiology 2003;229:659-69.

8. Cheung Y'T, Sabin ND, Reddick WE, Bhojwani D, Liu W, Brinkman TM, Glass JO, Hwang SN, Srivastava D, Pui CH, Robison LL, Hudson MM, Krull KR. Leukoencephalopathy and long-term neurobehavioural, neurocognitive, and brain imag-ing outcomes in survivors of childhood acute lymphoblastic leukaemia treated with chemotherapy: a longitudinal analysis. Lancet Haematol 2016;3:e456-66.

9. Hu Z, Zou D, Mai H, Yuan X, Wang L, Li Y, Liao J, Liu L, Liu G, Zeng H, Wen F. Altered brain function in new onset childhood acute lymphoblastic leukemia before chemotherapy: A resting-state fMRI study. Brain Dev
2017;39:743-50.

10. Morioka S, Morimoto M, Yamada K, Hasegawa T, Morita T, Moroto M, Isoda K, Chiyonobu T, Imamura T, Nishimura A, Morimoto A, Hosoi H. Effects of chemotherapy on the brain in childhood: diffusion tensor imaging of subtle white matter damage. Neuroradiology 2013;55:1251-7.

11. van der Plas E, Spencer Noakes TL, Butcher DT, Weksberg R, Galin-Corini L, Wan-stall EA, Te P, Hopf L, Guger S, Spiegler BJ, Hitzler J, Schachar RJ, Ito S, Nieman BJ. Quantitative MRI outcomes in child and adolescent leukemia survivors: Evidence for global alterations in gray and white matter. Neuroimage Clin 2020;28:102428.

12. Hermoye L, Saint-Martin C, Cosnard G, Lee SK, Kim J, Nassogne MC, Menten R, Clapuyt P, Donohue PK, Hua K, Wakana S, Jiang H, van Zijl PC, Mori S. Pediatric diffusion tensor imaging: normal database and observation of the white matter matu-ration in early childhood. Neuroimage 2006;29:493-504.

13. Loh KB, Ramli N, Tan LK, Roziah M, Rahmat K, Ariffin H. Quantification of dif-fusion tensor imaging in normal white matter maturation of early childhood using an automated processing pipeline. Eur Radiol 2012;22:1413-26.

14. Knickmeyer RC, Gouttard S, Kang C, Evans D, Wilber K, Smith JK, Hamer RM, Lin W, Gerig G, Gilmore JH. A structural MRI study of human brain development from birth to 2 years. J Neurosci 2008;28:12176-82.

15. Ramli N, Lim CH, Rajagopal R, Tan LK, Seow P, Ariffin $H$. Assessing changes in microstructural integrity of white matter tracts in children with leukaemia following exposure to chemotherapy. Pediatr Radiol 2020;50:1277-83.

16. Kaatsch P. Epidemiology of childhood cancer. Cancer Treat Rev 2010;36:277-85.

17. De Cocker LJ, Tousseyn T, Van Calenbergh F, Uyttebroeck A, Demaerel P. Menin-geal leukemia in acute lymphoblastic leukemia revealed by an intracranial mass. J Neuroradiol 2012;39:130-2.

18. Muthu V, Kumar S, Prakash G, Sharma P, Varma S. Image diagnosis: Weber syn-drome: a rare presentation of acute leukemia-a case report and review of the literature. Perm J 2015;19:83-5.

19. Hunger SP, Mullighan CG. Acute Lymphoblastic Leukemia in Children. N Engl J Med 2015;373:1541-52.

20. Cheung YT, Khan RB, Liu W, Brinkman TM, Edelmann MN, Reddick WE, Pei D, Panoskaltsis-Mortari A, Srivastava D, Cheng C, Robison LL, Hudson MM, Pui CH, Krull KR. Association of Cerebrospinal Fluid 
Biomarkers of Central Nervous System Injury With Neurocognitive and Brain Imaging Outcomes in Children Receiving Chemotherapy for Acute Lymphoblastic Leukemia. JAMA Oncol 2018;4:e180089.

21. van der Knaap MS, van der Grond J, van Rijen PC, Faber JA, Valk J, Willemse K. Age-dependent changes in localized proton and phosphorus MR spectroscopy of the brain. Radiology 1990;176:509-15.

22. Kreis R, Ernst T, Ross BD. Development of the human brain: in vivo quantification of metabolite and water content with proton magnetic resonance spectroscopy. Magn Reson Med 1993;30:424-37.

23. Pääkkö E, Harila-Saari A, Vanionpää L, Himanen S, Pyhtinen J, Lanning M. White matter changes on MRI during treatment in children with acute lymphoblastic leuke-mia: correlation with neuropsychological findings. Med Pediatr Oncol 2000;35:456-61.

24. Cecil KM, Hills EC, Sandel ME, Smith DH, McIntosh TK, Mannon LJ, Sinson GP, Bagley LJ, Grossman RI, Lenkinski RE. Proton magnetic resonance spectroscopy for detection of axonal injury in the splenium of the corpus callosum of brain-injured pa-tients. J Neurosurg 1998;88:795-801.

25. Duyn JH, Gillen J, Sobering G, van Zijl PC, Moonen CT. Multisection proton MR spectroscopic imaging of the brain. Radiology 1993;188:277-82.

26. Aukema EJ, Caan MW, Oudhuis N, Majoie CB, Vos FM, Reneman L, Last BF, Grootenhuis MA, Schoutenvan Meeteren AY. White matter fractional anisotropy correlates with speed of processing and motor speed in young childhood cancer sur-vivors. Int J Radiat Oncol Biol Phys 2009;74:837-43.

27. Schulte T, Sullivan EV, Müller-Oehring EM, Adalsteinsson E, Pfefferbaum A. Cor-pus callosal microstructural integrity influences interhemispheric processing: a diffusion tensor imaging study. Cereb Cortex 2005;15:1384-92.
28. Barkovich AJ, Kjos BO, Jackson DE Jr, Norman D. Normal maturation of the neona-tal and infant brain: MR imaging at 1.5 T. Radiology 1988;166:173-80.

29. Mukherjee P, Miller JH, Shimony JS, Philip JV, Nehra D, Snyder AZ, Conturo TE, Neil JJ, McKinstry RC. Diffusion-tensor MR imaging of gray and white matter development during normal human brain maturation. AJNR Am J Neuroradiol 2002;23:1445-56.

30. Salat DH, Lee SY, Yu P, et al. DTI in Development and Aging. Diffus MRI 2009:205-36.

31. Jacola LM, Krull KR, Pui CH, Pei D, Cheng C, Reddick WE, Conklin HM. Longi-tudinal Assessment of Neurocognitive Outcomes in Survivors of Childhood Acute Lymphoblastic Leukemia Treated on a Contemporary Chemotherapy Protocol. J Clin Oncol 2016;34:1239-47.

32. Edelmann MN, Krull KR, Liu W, Glass JO, Ji Q, Ogg RJ, Sabin ND, Srivastava DK, Robison LL, Hudson MM, Reddick WE. Diffusion tensor imaging and neurocognition in survivors of childhood acute lymphoblastic leukaemia. Brain 2014;137:2973-83.

33. Khong PL, Leung LH, Fung AS, Fong DY, Qiu D, Kwong DL, Ooi GC, McAlonan G, Cao G, Chan GC. White matter anisotropy in post-treatment childhood cancer survivors: preliminary evidence of association with neurocognitive function. J Clin Oncol 2006;24:884-90.

34. Porto L, Preibisch C, Hattingen E, Bartels M, Lehrnbecher T, Dewitz R, Zanella F, Good C, Lanfermann H, DuMesnil R, Kieslich M. Voxel-based morphometry and diffusion-tensor MR imaging of the brain in long-term survivors of childhood leuke-mia. Eur Radiol 2008;18:2691-700.

35. Lim YJ, Kim HJ, Lee YJ, Seol IJ, Lee YH. Clinical features of encephalopathy in children with cancer requiring cranial magnetic resonance imaging. Pediatr Neurol 2011;44:433-8.
Cite this article as: Wei K, Liang Y, Yang B, Liu L, Cao W, Li T, Wang R. An observational MRI study of methotrexatetreated children with acute lymphoblastic leukemia in remission and subtle cognitive decline. Quant Imaging Med Surg 2022;12(4):2474-2486. doi: 10.21037/qims-21-748 


\section{Clinical risk classification}

Participants were categorized into three groups, namely: (I) standard risk ( $\mathrm{SR}, \mathrm{n}=14)$; intermediate risk (IR, $\mathrm{n}=13$ ); and high risk (HR, $n=3$ ), based on age at first diagnosis, total number of peripheral white blood cells (WBC), response to 7-day prednisone treatment, the translocation of $\mathrm{t}(4 ; 11)$ and $t(9 ; 22)$, fusion gene detection (MLL/AF4 and BCR/ $\mathrm{ABL}$ ), and bone marrow remission status on days 15 and 33. Patients with (i) SR met the following criteria: good response to 7-day prednisone treatment; peripheral blood immature cells on day $8<1.0 \times 10^{9} / \mathrm{L}$; age $\geq 1$ year and $<7$ years old; $\mathrm{WBC}<20 \times 10^{9} / \mathrm{L}$; induction chemotherapy bone marrow M1 (primitive lymphocytes + naive lymphocytes) $<5 \%$ or M2 (primitive lymphocytes + naive lymphocytes) between $5 \%$ and $25 \%$ on day 15 ; and bone marrow M1 on day 33 of induction chemotherapy. Patients with (ii) IR met the following criteria: a good prednisone response; peripheral blood immature cells $<1.0 \times 10^{9} / \mathrm{L}$ on day 8 ; age $<1$ year or $\geq 7$ years; $W B C \geq 20 \times 10^{9} / \mathrm{L}$; and induction chemotherapy patients with bone marrow M3 (primitive lymphocytes + naive lymphocytes) $>25 \%$ on day 15 and M1 on day 33. Patients with (iii) HR met at least one of the following criteria: not SR or receiving induction chemotherapy; bone marrow M3 on day 15; a poor prednisone response; peripheral blood naive cells $\geq 1.0 \times 10^{9} / \mathrm{L}$ on day 8 ; bone marrow M2 or M3 on day 33; or a $\mathrm{t}(4 ; 11)(\mathrm{MLL} / \mathrm{AF} 4)$ or $\mathrm{t}(9: 22)$ (BCR/ABL) abnormality.

\section{Clinical treatment}

Children with ALL were treated according to the German BFM2002 protocol for acute lymphoblastic leukemia (ALL) based on their risk group (SR, IR or HR). The induction therapy protocol was as follows: day 1 to day 7 prednisone test - prednisone $60 \mathrm{mg} /\left(\mathrm{m}^{2}\right.$.d), 3 times orally; day 8 to day 28 dexamethasone $6 \mathrm{mg} /\left(\mathrm{m}^{2} . \mathrm{d}\right)$ taken orally 3 times per day. After day 29 , the dose of dexamethasone was gradually reduced; it was halved every 3 days, to be taken in the morning, with the dose reduction to be completed on day 9. Vincristine (VCR): $1.5 \mathrm{mg} /\left(\mathrm{m}^{2} . \mathrm{d}\right)(\ngtr 2 \mathrm{mg} / \mathrm{time})$, intravenous bolus on day 8 , day 15 , day 22 , and day 29 . DNR $30 \mathrm{mg} /\left(\mathrm{m}^{2} . \mathrm{d}\right)$, intravenous bolus maintenance $(\mathrm{PI})>1 \mathrm{~h}$; for SR-ALL, $\mathrm{DNR} \times 2$, on day 8 and day 15 ; for IR-ALL, DNR $\times 4$, on day 9 , day 15 , day 22, and day 29. L-ASP: $5000 \mathrm{U} /\left(\mathrm{m}^{2} . \mathrm{d}\right)$, PI $>1 \mathrm{~h}$, on day 36 and day 64. 6-MP: $60 \mathrm{mg} /\left(\mathrm{m}^{2} . \mathrm{d}\right)$, orally, on day 36 to day 63 (for 28 days total), taken at night on an empty stomach. Ara-C: $75 \mathrm{mg} /\left(\mathrm{m}^{2} . \mathrm{d}\right)$, intravenous bolus, administered in 4 parts, for 4 days each time, on day 38 to day 41 , on day 45 to day 48 , on day 52 to day 55 , and on day 59 to day 62 . Consolidation treatment: 6-MP: $25 \mathrm{mg} /\left(\mathrm{m}^{2} . \mathrm{d}\right)$, orally, day 1 to day 56 , taken at night on an empty stomach. Medium dose methotrexate (MTX): $200 \mathrm{mg} /\left(\mathrm{m}^{2} . \mathrm{d}\right)$, PI $24 \mathrm{~h}$, once every 14 days, 4 times in total (day 8 , day 22, day 36 , and day 50 ); $1 / 10$ of the total $\left.\left(200 \mathrm{mg} / \mathrm{m}^{2}\right)\right)$ to be administered for the loading dose. Intravenous drip over $30 \mathrm{~min}, 9 / 10$ of the total $\left(1800 \mathrm{mg} / \mathrm{m}^{2}\right)$ PI $23.5 \mathrm{~h}$. Reinduction therapy: dexamethasone $-8 \mathrm{mg} /\left(\mathrm{m}^{2} . \mathrm{d}\right)$, orally, 3 times, on day 1 to day 21 . From day 22 onwards, the dose was gradually reduced to stop the drug; it was halved every 3 days, to be taken in the morning. VCR: $1.5 \mathrm{mg} /\left(\mathrm{m}^{2} . \mathrm{d}\right)$, intravenous bolus ( $\ngtr 2 \mathrm{mg} /$ time), on day 8 , day 15 , day 22 , and day 29 . DOX: $30 \mathrm{mg} /\left(\mathrm{m}^{2} . \mathrm{d}\right)$, intravenous bolus, over $1 \mathrm{~h}$, on day 8, day 15 , day 22 , and day 29. L-ASP: $10,000 \mathrm{U} /\left(\mathrm{m}^{2} . \mathrm{d}\right)$, PI $>1$ h, on day 8 , day 11 , day 15 , and day 18. CTX: $1 \mathrm{~g} /\left(\mathrm{m}^{2} . \mathrm{d}\right)$, PI $>1 \mathrm{~h}$, on day 36. Ara-C: $75 \mathrm{mg} /\left(\mathrm{m}^{2} . \mathrm{d}\right)$, intravenous bolus, with 2 courses of treatment, each course for 4 days, on day 38 to day 41 and on day 45 to day 48. 6-MP: $60 \mathrm{mg} /$ $\left(\mathrm{m}^{2} . \mathrm{d}\right)$, on day 36 to day 49 (14 days), taken orally at night, in a fasted state, with milk. Consolidation treatment was mainly given through intravenous or intrathecal injection of various doses of methotrexate (IV-MTX and IT-MTX, respectively). After bone marrow aspiration was carried out, no obvious abnormality was found by routine MRI examination. 